\title{
Primary Care Physicians' Perceived Barriers to Nephrology Referral and Co-management of Patients with CKD: a Qualitative Study
}

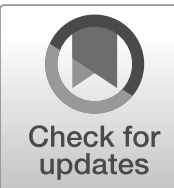

\author{
Raquel C. Greer, MD, MHS ${ }^{1,2}$, Yang Liu, MPH ${ }^{1,3}$, Kerri Cavanaugh, MD, $M H S^{4,5}$, \\ Clarissa Jonas Diamantidis, $M D, M H S^{6}$, Michelle M. Estrella, MD, $M H S^{7}$, C. John Sperati, $M D, M H S^{8}$, \\ Sandeep Soman, $M D^{9}$, Khaled Abdel-Kader, $M D, M S^{4,5}$, Varun Agrawal, $M D^{10}$, \\ Laura C. Plantinga, $P h D^{17,12}$, Jane O. Schell, $M D^{13}$, James F. Simon, $\mathrm{MD}^{14}$, \\ Joseph A. Vassalotti, MD ${ }^{15,16}$, Bernard G. Jaar, MD, MPH ${ }^{1,8,17,18}$, and Michael J. Choi, MD ${ }^{8}$ On behalf \\ of the National Kidney Foundation Education Committee
}

\begin{abstract}
'The Welch Center for Prevention, Epidemiology, and Clinical Research, Baltimore, MD, USA; ²Division of General Internal Medicine, Johns Hopkins University, Baltimore, MD, USA; ${ }^{3}$ Johns Hopkins Medicine International, Johns Hopkins Medical Institutions, Baltimore, MD, USA; ${ }^{4}$ Vanderbilt Center for Kidney Disease, Vanderbilt University Medical Center, Nashville, TN, USA; ${ }^{5}$ The Division of Nephrology, Vanderbilt University Medical Center, Nashville, TN, USA; ${ }^{6}$ Divisions of General Internal Medicine and Nephrology, Duke University School of Medicine, Durham, NC, USA; ${ }^{1}$ Kidney Health Research Collaborative, Department of Medicine, Kidney Health Research Collaborative, Department of Medicine, University of California, San Francisco and San Francisco VA Health Care System, San Francisco, CA, USA; ${ }^{2}$ Division of Nephrology, Department of Medicine, Johns Hopkins University School of Medicine, Baltimore, MD, USA; ${ }^{9}$ Division of Nephrology and Hypertension, Henry Ford Hospital, Detroit, MI, USA; ${ }^{10}$ Division of Nephrology and Hypertension, University of Vermont, Burlington, VT, USA; ${ }^{1}$ Department of Medicine, Emory University, Atlanta, GA, USA;

${ }^{12}$ Department of Epidemiology, Emory University, Atlanta, GA, USA; ${ }^{13}$ Section of Palliative Care and Medical Ethics, Renal-Electrolyte University of Pittsburgh School of Medicine, University of Pittsburgh Medical Center, Pittsburgh, PA, USA; ${ }^{14}$ Department of Nephrology and Hypertension, Glickman Urologic and Kidney Institute, Cleveland Clinic, Cleveland, OH, USA; ${ }^{15}$ National Kidney Foundation, New York, NY, USA; ${ }^{16}$ Icahn School of Medicine at Mount Sinai, New York, NY, USA; ${ }^{17}$ Nephrology Center of Maryland, Baltimore, MD, USA; ${ }^{18}$ The Welch Center for Prevention,

Epidemiology, and Clinical Research Institutions, Baltimore, MD, USA.
\end{abstract}

BACKGROUND: Effective co-management of patients with chronic kidney disease (CKD) between primary care physicians (PCPs) and nephrologists is increasingly recognized as a key strategy to ensure the delivery of efficient and high-quality CKD care. However, the co-management of patients with CKD remains suboptimal.

OBJECTIVE: We aimed to identify PCPs' perceptions of key barriers and facilitators to effective co-management of patients with CKD at the PCP-nephrology interface.

STUDY DESIGN: Qualitative study

SETTING AND PARTICIPANTS: Community-based PCPs in four US cities: Baltimore, MD; St. Louis, MO; Raleigh, NC; and San Francisco, CA

APPROACH: We conducted four focus groups of PCPs. Two members of the research team coded transcribed audio-recorded interviews and identified major themes.

KEY RESULTS: Most of the 32 PCPs (59\% internists and $41 \%$ family physicians) had been in practice for $>10$ years (97\%), spent $\geq 80 \%$ of their time in clinical care (94\%), and practiced in private (69\%) or multispecialty group practice (16\%) settings. PCPs most commonly identified barriers to effective co-management of patients with CKD focused on difficulty developing working partnerships with nephrologists, including (1) lack of timely adequate information

Prior Presentation The findings of the study were previously presented at the National Kidney Foundation Spring Clinical Meeting. Orlando, FL. April 2017.

Received May 17, 2018

Revised December 11, 2018

Accepted February 20, 2019

Published online April 16, 2019 exchange (e.g., consult note not received or CKD care plan unclear); (2) unclear roles and responsibilities between PCPs and nephrologists; and (3) limited access to nephrologists (e.g., unable to obtain timely consultations or easily contact nephrologists with concerns). PCPs expressed a desire for "better communication tools" (e.g., shared electronic medical record) and clear CKD care plans to facilitate improved PCP-nephrology collaboration.

CONCLUSIONS: Interventions facilitating timely adequate information exchange, clear delineation of roles and responsibilities between PCPs and nephrologists, and greater access to specialist advice may improve the co-management of patients with CKD.

KEY WORDS: chronic kidney disease; primary care; nephrology; comanagement; qualitative research.

$\mathrm{J}$ Gen Intern Med 34(7):1228-35

DOI: $10.1007 / \mathrm{s} 11606-019-04975-\mathrm{y}$

(c) Society of General Internal Medicine 2019

\section{INTRODUCTION}

Chronic kidney disease (CKD) is an important public health problem that is associated with considerable morbidity, mortality, and health care costs. ${ }^{1,2}$ Patients with CKD often have multiple co-morbid conditions that are managed by multiple providers across various settings, often without clear communication between providers. ${ }^{3}$ This decentralized system can lead to fragmented care that adversely affects patients' health 
and the provision of efficient and high-quality care to mitigate patients' risks of CKD progression (e.g., delayed care, unnecessary and/or inappropriate tests, or medical errors). ${ }^{4}$

Effective co-management of patients with CKD is increasingly recognized as a key strategy to ensure the delivery of efficient, high-quality, and safe care and has been shown to contribute to greater rates of assessment and treatment of patients' CKD risks. ${ }^{4-9}$ Primary care and nephrology providers are two key participants responsible for managing patients' risks for CKD progression and CKD-related complications, and desire improved co-management of patients with CKD. ${ }^{10}$ While previous studies have characterized the quality and barriers to coordination of care between primary care physicians (PCPs) and specialists, ${ }^{11,12}$ the barriers to comanagement of patients with CKD specifically between primary care and nephrology providers have not been wellcharacterized or explored in-depth, particularly among US providers, and the presence of unique barriers to integrated care at the nephrology-primary care interface is unknown.

The goal of this study was to identify PCPs' perceptions of key factors contributing to suboptimal co-management of patients with CKD among a diverse group of PCPs in the United States of America.

\section{METHODS}

We conducted four focus groups with a total of 32 PCPs $(8$ PCPs per group) to identify their (1) perceived barriers to successful nephrology referral and effective co-management of patients with CKD with nephrologists and (2) views of potential tools and resources which could improve the comanaged care of CKD.

\section{Study Participants}

A sample of PCPs from four US cities (Baltimore, MD; St. Louis, MO; Raleigh, NC; and San Francisco, CA) was recruited by Baltimore Research to participate in the focus groups. Baltimore Research recruits providers from an internal list of physicians who have previously participated in research studies and uses a variety of methods such as phone, fax, email, and/or referral to reach out to potential participants. To be eligible to participate in the study, respondents needed to be in active clinical practice in primary care (including practice at least one half day per week and care for $>40$ patients monthly), spend the majority of their clinical time delivering outpatient primary care, and care for patients with CKD. To obtain a study sample representing PCPs from different specialties (i.e., family practice and internal medicine); practice settings (i.e., community-based and university/ medical school-based practice); and genders (i.e., male and female) and PCPs who were racially/ethnically diverse (i.e., included providers identifying as Black/African American, Hispanic/ Latino, Native American, Native Hawaiian or Other Pacific Islander), we set quotas for the minimum number of PCPs that should be represented in each of above specified groups. Written consent was obtained from all participants, who received a monetary incentive for their participation. The Johns Hopkins Medicine Institutional Review Board approved the study protocol.

\section{Data Collection}

The focus groups were conducted between April and June 2015. Each 90-minute focus group session began with a selfadministered questionnaire to obtain physicians' demographic and practice characteristics, and to assess the physicians' comfort with the management and education of their patients with CKD. An investigator (RCG), a general internist and health services researcher with expertise in qualitative methods, ${ }^{13-15}$ conducted all sessions using a semi-structured question guide developed by the National Kidney Foundation Education Committee. The question guide consisted of open-ended questions with the goal of identifying PCPs' perceived barriers and facilitators to nephrology referral and co-management of patients with CKD (Textbox). Detailed notes were taken during the focus group session and the focus group guide (i.e., questions and prompts) for subsequent groups was modified in an iterative fashion to address emerging themes. Data collection continued until thematic saturation was achieved (i.e., no new themes identified). The focus groups were also audiotaped and transcribed verbatim for thematic content analysis. ${ }^{16}$

\section{Textbox Focus group questions}

- Describe your experience with referring a patient to a nephrologist both during the referral process and collaborating with the nephrologist. - What challenges do you experience with referring a patient to nephrology? What challenges do you experience in collaborating with nephrology?

- Please describe resources, tools, or features of your practice that make it easier for you to refer a patient to nephrology or collaborate with your patients' nephrologist?

- What would you find helpful in referring your patients to a nephrologist or collaborating with your patients' nephrologist?

\section{Analysis}

We used content analysis to analyze the focus group discussions. Two members (RCG, YL) of the research team independently reviewed the initial two focus group transcripts to iteratively develop a coding scheme using an inductive approach (i.e., codes emerged from the text and were not predefined) that represented the relevant concepts addressed during the focus group discussions. ${ }^{16,17}$ The codes were descriptive labels assigned to text segments of varying sizes that described the challenges PCPs face in co-managing patients with CKD with nephrologists (e.g., lack of timely information exchange or difficulty scheduling patients with nephrologists in a timely fashion), as well as their views on what would be helpful in improving the co-managed care (e.g., ability to more easily contact nephrologist). The two investigators reviewed and jointly agreed on the final coding scheme that they then applied to all of the transcripts. Any emergent codes were reviewed and subsequently added to the final coding scheme. Similar codes were then grouped together 
into broader conceptual categories to develop a final list of major themes and subthemes regarding PCPs' perceptions of barriers and facilitators to effective co-management of patients with CKD. ATLAS.ti version 5.0. (ATLAS.ti GmbH, Berlin, Germany) was used for data management.

\section{RESULTS}

A total of 32 PCPs (59\% internists and $41 \%$ family physicians) participated in the four focus group sessions. The participating physicians were $41 \%$ female, $66 \%$ White, $6 \%$ African American, $6 \%$ Hispanic, and $16 \%$ Asian. Most had been in practice for $>10$ years $(97 \%)$, spent $\geq 80 \%$ of their time in clinical care (94\%), and practiced in a private (69\%) or multispecialty group practice (16\%) settings. All physicians reported using electronic medical records (EMRs) entirely or in part, and 54\% reported following CKD guidelines (Table 1).

\section{PCPs' Perceived Barriers to Nephrology Referral and Co-management}

PCPs identified five key barriers to timely nephrology referral and effective co-management of patients with CKD (Table 2). Lack of Timely and Adequate Information Exchange. Many PCPs reported not receiving consultative notes from nephrologists at all or in a timely fashion. This often resulted

Table 1 Participant Characteristics $(n=32)$

\begin{tabular}{|c|c|}
\hline & Total \\
\hline Age, mean years (SD) & $53(8)$ \\
\hline Female, $n(\%)$ & $13(41)$ \\
\hline \multicolumn{2}{|l|}{ Race/ethnicity, $n(\%)$} \\
\hline White & $21(66)$ \\
\hline Black/African American & $2(6)$ \\
\hline Hispanic/Latino & $2(6)$ \\
\hline Asian & $5(16)$ \\
\hline Other & $2(6)$ \\
\hline \multicolumn{2}{|l|}{ Medical specialty, $n(\%)$} \\
\hline Internal medicine & $19(59)$ \\
\hline Family practice & $13(41)$ \\
\hline \multicolumn{2}{|l|}{ Training, $n(\%)$} \\
\hline Doctor of Medicine (MD) & $30(94)$ \\
\hline Doctor of Osteopathic Medicine (DO) & $2(6)$ \\
\hline \multicolumn{2}{|l|}{ Number of years in practice, $n(\%)$} \\
\hline$<10$ years & $1(3)$ \\
\hline $11-15$ years & $8(25)$ \\
\hline$>15$ years & $23(72)$ \\
\hline Percent clinical time, median (IQR) & $98(88-100)$ \\
\hline \multicolumn{2}{|l|}{ Practice setting, $n(\%)$} \\
\hline Solo private practice & $10(31)$ \\
\hline Single specialty group private practice & $12(38)$ \\
\hline Multispecialty group practice & $5(16)$ \\
\hline University hospital or medical school & $2(6)$ \\
\hline Community hospital & $2(6)$ \\
\hline Government health care facility & $1(3)$ \\
\hline \multicolumn{2}{|l|}{ Number of patients per week } \\
\hline$\leq 100$ & $20(62)$ \\
\hline$>100$ & $12(38)$ \\
\hline \multicolumn{2}{|l|}{ Number of CKD patients per week } \\
\hline$\leq 10$ & $10(31)$ \\
\hline$>10$ & $22(69)$ \\
\hline $100 \%$ electronic medical record use, $n(\%)$ & $23(72)$ \\
\hline Reported following CKD guidelines, $n(\%)$ & $17(54)$ \\
\hline
\end{tabular}

$n$ number of participants, CKD chronic kidney disease in patients being the conduit for facilitating information exchange from the nephrologist to the PCP regarding the CKD care plan (e.g., medication changes, recommended timing of follow-up visits, and test results). A PCP stated,

...well the note back from the specialist, in this case the nephrologist, may or may not make it back to you....I've had biopsies done on these people and I'll see the patient and they say 'yeah they did x, y, z' and I'm like oh really? Then I'm the one looking like an idiot in front of them. They felt that "we [providers] are still working in our silos," and this fragmented health care system may contribute to medication errors and duplication of tests. A PCP stated,

I'll just draw it [labs] myself because I can't get [them]. Because I mean you can only have so much time in a day to chase something.Information exchange between nephrologists and PCPs was noted to be even more challenging among patients on dialysis. A PCP commented,

These are often our most complex patients and sickest patients [dialysis patients], yet there's this black hole they fall into and when they pop out you have no idea what's been going on with them.PCPs also raised concerns regarding the comprehensiveness of the consultative notes, which sometimes lacked clear plans for various

Table 2 Primary Care Providers' Perceived Barriers to Nephrology Referral and Co-management

\begin{tabular}{ll}
\hline \hline Major themes & Subthemes \\
\hline $\begin{array}{l}\text { Barriers } \\
\text { Lack of timely adequate } \\
\text { information exchange }\end{array}$ & $\begin{array}{l}\text { Lack of information exchange } \\
\text { Lack of comprehensive } \\
\text { consultative notes }\end{array}$ \\
Limited access to nephrologists & $\begin{array}{l}\text { Unable to obtain timely } \\
\text { consultations }\end{array}$ \\
& $\begin{array}{l}\text { Unable to easily contact } \\
\text { nephrologist }\end{array}$ \\
Unclear delineation of roles and & $\begin{array}{l}\text { Providers' lack of clarity } \\
\text { regarding roles }\end{array}$ \\
responsibilities & $\begin{array}{l}\text { Expectations of referral not met } \\
\text { Patients unclear of nephrology } \\
\text { role }\end{array}$ \\
Poor working relationships with & $\begin{array}{l}\text { Difficulty establishing good } \\
\text { consultative relationship }\end{array}$ \\
nephrologist & $\begin{array}{l}\text { Perceived lack of respect from } \\
\text { specialists }\end{array}$ \\
Patients sometimes prefer PCP \\
Patients' lack of trust/established & handle most care \\
relationship with nephrologists & $\begin{array}{l}\text { Patients' lack of trust with } \\
\text { nephrologists }\end{array}$ \\
&
\end{tabular}

Facilitator

Refer to nephrologists with whom you have a good consultative relationship

Timely adequate information exchange
Refer to small number of specialists Nephrologists easily accessible Comprehensive consultative notes

Timely information exchange (i.e., shared EMR) PCP ensures nephrologist has necessary information 
aspects of CKD care. This was particularly concerning for PCPs who were less familiar with the commonly prescribed medications in CKD. A PCP commented,

...some of the medications that the nephrologists use I don't use. I mean I don't start [the patient] on it, but when they refer back I don't know how long I'm supposed to keep them on the medications or is it safe. The last thing that as a primary care physician I want to do is hurt my patient. By not knowing that oh, you shouldn't have kept them on that, well I didn't know that. You didn't tell me. There's no note.Some PCPs felt nephrologists may fail to recognize that PCPs may not be familiar with all aspects of the management of advanced CKD and CKD-related complications, such as metabolic acidosis.

Limited Access to Nephrologists. Some PCPs described challenges obtaining nephrology consultations in a timely fashion due to a shortage of nephrologists in their area and/ or insurance restrictions. A PCP noted,

There is still a shortage of nephrologists. Just getting in to see one sometimes, it can be a matter of a couple of months sometimes, especially if you want something acutely.Another provider commented,

...you're going to have some access issues, ... because they[insurance carriers] just haven't contracted with enough nephrologists or the nephrologists are not geographically in a convenient place for that patient...Many PCPs also noted their frustration with being unable to easily contact nephrologists with issues or concerns regarding patients' CKD care, which may contribute to significant delays in patient care.

I'm always calling saying, if you just tell me send me an e-mail because I have to call you each time...

Unclear Delineation of Roles and Responsibilities. Most PCPs also reported a lack of clarity regarding roles and responsibilities. They felt that the responsible party for managing each aspect of CKD was not explicitly documented in the nephrologists' notes. A PCP stated,

And I don't feel like the nephrologists do a very good job of like sending [a consult note]- - to me to say I'm following her, you're following her, is somebody following her.Some PCPs also described that, despite providing the nephrology team with appropriate and adequate information regarding the reason for referral, their referral question was not always addressed by the nephrologist. A PCP commented,

I give them the referral, they [the patient] may have the same piece of paper I sent electronically to the doctor, and I don't know if they read my question because it doesn't get answered. And it's very frustrating...In addition, some PCPs were frustrated by occasions when referring a patient to a nephrologist for either a one-time consultation and/or to establish a comanagement relationship, the nephrologist would assume the primary care role of the patient. This could include subsequently making referrals to other specialists for secondary diagnoses without notifying the PCP. A PCP reported,

... the common complaint that I would hear is once you refer your patient [there] that patient disappears.PCPs also described that patients were often unclear of the role of the nephrologist, so it was often difficult to have patients keep follow-up nephrology appointments.

...they [patients] also spent $\$ 60$ and they're like why don't you just do that? He [the nephrologist] didn't do anything that you didn't do.

Poor Working Relationships with Nephrologists. Some PCPs described difficultly establishing new effective consultative relationships with nephrologists, including finding nephrologists who were accessible to consultations and urgent questions, provided them with informative consult notes in a timely fashion, and were respectful of their questions. For example, some described receiving complaints about the timing of the referrals, both for referring too early and/or too late. Some also commented that nephrologists did not know how to relate to primary care, particularly when collaborating with nephrologists at academic medical centers. One PCP commented,

...they just don't get the relationship. They really don't understand it... you guys don't even say thank you. I'm referring my patient to you. You do not give me the third degree or say what I have to do....if we're going to jump through hoops [for you] to see my patient then okay, I'll send my patient somewhere else. You can't do that in private practice so the nephrologist or any specialist is not going to do that. They're going to send a note, they're going to say thank you for sending your very lovely [patient]...Another commented,

...but I can tell you that a lot of times even though I'm extremely well-trained, [to the nephrologist] I'm [the] stupid primary care doctor who doesn't seem to know anything...

Patients' Lack of Trust and/or Established Relationship with Nephrologists. Some PCPs described that patients' lack of trust or an established relationship with their nephrology providers was a barrier to effective co-management. Patients' 
often preferred to see their PCP rather than the nephrologist or to get their PCPs' approval regarding medication changes, which resulted in delays in care. A PCP reported,

[The patient states] I don't want to go any place if I can just stay here.Another described,

And a lot of patients will just ignore what the specialist says because they trust their primary care doctor, and so you find out...six months later that they were supposed to be taking something different as far as the nephrologist was concerned.

\section{PCPs' Perceived Facilitators to Nephrology Referral and Co-management}

PCPs described referring to a small number of nephrologists with whom they had a good consultative relationship, specifically those providers who were easily accessible, offered timely appointments, and provided comprehensive care plans. A PCP commented,

... if I'm really worried about something, I text the nephrologist I know real well and say...this is what's going on, it's in the record, and they get in. They felt that prompt, adequate information exchange was the key to improving co-management of patients with CKD, which was facilitated by a shared EMR. A provider commented,

I think for me the most important thing would be just having a shared EMR where you can just look up that encounter very quickly.PCPs also agreed that the consultative notes should have a clear and thorough CKD care plan. For example, if the patient has metabolic acidosis, the note should include the diagnosis, the treatment, and the management goal. A PCP stated,

Well the first thing is send us the notes back on these patients and clarify is this medication meant to be lifelong, one month, two weeks, or whatever. Specify some type of treatment plan...They also noted that bidirectional exchange of information was important.

\section{DISCUSSION}

In this qualitative study of community-based PCPs, we identified several key barriers to effective co-management between PCPs and nephrologists for patients with CKD, including lack of timely adequate information exchange, unclear delineation of roles and responsibilities, limited access to nephrologists, poor working relationships with nephrologists, and patients' lack of established relationships with nephrologists. While the importance of effective partnerships between primary care and nephrology is well-recognized, few studies have explored indepth PCPs' experience with co-managing patients with nephrologists. ${ }^{8,10,13,18}$ This study identifies key modifiable barriers that exist to effective co-management of patients with $\mathrm{CKD}$. The findings from this study can inform interventions to improve the efficiency, quality, and safety of co-managed care of CKD, and are also generalizable to other chronic illnesses.

The PCPs in our study identified poor information exchange as a key barrier to co-management of patients with nephrologists; this is consistent with findings of prior studies assessing the co-management of patients between PCPs and specialists. ${ }^{11,13,19,20}$ While many specialty and primary care practices have EMRs, many EMRs lack interoperability and relatively few real-time health information exchanges currently exist. These limitations prevent providers from efficiently sharing information across health systems. This, in turn, creates a barrier for PCPs and specialists to easily communicate with one another and to have the necessary information to facilitate clinical decision-making during the patient visit. The development of EMRs with interoperability and robust health information exchange infrastructure and supports to facilitate the exchange of key information in a clear and concise format may allow providers to easily access and reconcile information (e.g., visit notes, medication lists, and test results) from other health care sites. This could have tremendous potential in improving coordination of care, as well as patient care and outcomes. ${ }^{21-24}$ In the absence of these tools, efforts are needed to increase providers' adoption and efficient use of existing strategies to enhance timely information exchange, such as the integration of collaboration tools within EMRs.

The findings in our study also reinforce prior work emphasizing that effective integration of primary and specialty care requires that there be a shared understanding of whom is responsible for different aspects of care. This is particularly important for $\mathrm{CKD}$, where there can be significant overlap in roles and responsibilities between PCPs and nephrologists for various aspects of CKD care (i.e., hypertension or lipid management). ${ }^{8,13,18,23,25}$ In addition, PCPs need better access to specialists' advice. There are several promising approaches that directly address PCPs' desire for role clarity and timely communication with specialists, including electronic consultations (i.e., e-consults or e-referrals), the use of referral and consult protocols/templates, and care coordination agreements. ${ }^{426-29}$ Many of these tools have been applied to CKD.

Electronic consultation is a tool within the EMR in which PCPs can pose a clinical question to specialists, who then can answer the consult question based on chart review, request more information, or approve a face-to-face specialty clinic appointment. This approach has been increasingly adopted by health care systems and has been shown to improve the timeliness and efficiency of specialty care. ${ }^{26,30-32}$ In the US Veterans Health Administration, e-consults are heavily utilized and its success is largely due to its integration within the 
existing EMR which made it convenient and easy for providers to use. Also, each specialty had the flexibility to determine how it would triage and assign responsibility for submitted e-consults. ${ }^{33}$ In the absence of electronic systems, the Renal Physicians Association has also developed nephrology referral protocols which have been shown to improve the comanaged CKD, including (1) referring physician templates to help clarify the reason for referral and requested consultation relationship (i.e., opinion only vs. co-management) and (2) post-consult letter templates to help facilitate communication of key CKD care plans and to clarify who is responsible for the different aspects of CKD care. ${ }^{8,34}$ Both electronic consultations and referral protocols have generally been well-received by PCPs and are felt to improve co-management of patients with specialists; however, there is some concern on the part of both PCPs and specialists related to increased workload burden. ${ }^{8,27,35}$ More research is needed to evaluate the effectiveness of these approaches in improving co-management, clinical outcomes, and cost of care across diverse practice settings.

Care coordination agreements (CCAs), which are written agreements to clearly define roles and responsibilities of PCPs and specialists, are another approach to help address PCPs' perceived barriers to co-management. These agreements formalize how physicians work together at all phases of their interaction, including the (1) pre-consult phase (i.e., PCP provides appropriate and accurate information and prepares patients, nephrologist review and triage); (2) consult (PCP clearly states reason for referral, nephrologist provides appropriate and adequate information in a timely fashion); and (3) co-management (both parties agree on who manages different aspects of CKD care, exchange notes in a timely fashion, and notify each other of changes in patients' health status, hospitalizations, etc.). ${ }^{36} \mathrm{CCAs}$, endorsed by the American College of Physicians, ${ }^{37}$ have been adopted by a diverse group of primary care practices to improve the co-management of patients with specialists, including the co-management of patients with $\mathrm{CKD}{ }^{38}$ While evidence is limited regarding the effectiveness of CCAs in improving care coordination and patient outcomes, those who have implemented CCAs report improvement in care delivery. ${ }^{36}$

Improving PCPs' knowledge of CKD guidelines may also improve timeliness of nephrology referrals and PCPnephrology collaboration. Over half of the PCPs in our study endorsed following any guidelines related to CKD; however, few providers specifically cited referring to CKD-specific guidelines such as Kidney Disease Improving Global Outcomes (KDIGO) Clinical Practice Guidelines for the Evaluation and Management of $\mathrm{CKD}^{6}$ or the National Kidney Foundation Kidney Disease Outcomes Quality Initiative (KDOQI). ${ }^{39}$ This finding is supported by our study as well as others, reporting PCPs' lack of awareness and familiarity with CKD guidelines. ${ }^{40,41}$ Efforts to promote greater PCP awareness and familiarity with CKD guideline may facilitate improved care coordination. $^{9}$
Some of the PCPs in our study did not feel respected when interacting with nephrologists. This is consistent with prior work describing PCPs' perceived lack of respect from specialists, a general negative attitude towards the discipline of primary care, and a lack of recognition of PCPs' clinical expertise. ${ }^{42}$ Poor working relationships with specialist may influence PCPs' referral decisions and negatively impact patient care. PCPs reported referring to nephrologists in which they had good consultative relationships (e.g., could easily access and received timely and adequate consultative notes). Efforts to promote referrals and strengthen relationships with specialists who are "good neighbors" (i.e., are easily accessible and send back a comprehensive consultative note in timely fashion) could also improve the integration of care for patients. ${ }^{4}$ In addition, greater emphasis on integrated care within medical education, training, and certification as well as the important role PCPs play in the health care system may better equip PCPs and specialists to deliver integrated care to their patients with CKD. ${ }^{4}$

Improving the co-management of patients between specialists and PCPs will require greater investment in care coordination activities. Activities related to coordination of care that take place outside the patient visit are not reimbursed. Similarly, physicians do not have sufficient staff or time to invest in coordination of care, and if they do, the provider bears the burden of the costs. The Veterans Health Administration is an example of a health system that has made substantial investments to improve the delivery of specialty care by implementing several initiatives that target multiple barriers to integrated care at the specialty-primary care interface, including (1) e-consults to improve PCPs' access to specialist advice; (2) Specialty Care Access Networks-Extension for Community Healthcare Outcomes (SCAN-ECHO), in which specialists lead multisite videoconferencing sessions with PCPs that include case-based learning and knowledge sharing to improve PCPs' clinical expertise in the specialty area; and (3) Specialty Care Neighborhoods (SCNs), team-based specialty care models for conditions such as heart failure. ${ }^{43}$ The evaluation of these strategies is currently ongoing. Payment reform and financial incentives to facilitate coordination of care (e.g., ACO, bundle payments) and greater adoption of multidisciplinary care approaches, such as the patient-centered medical home (e.g., provision of dedicated care coordination staff), could also have a dramatic impact on improving the receipt of coordinated and comprehensive care for patients with CKD and other chronic illnesses. ${ }^{4,25,37}$

The limitations of the study deserve mention. While we aimed to obtain the perspectives of a diverse set of PCPs, the barriers and facilitators identified in our study may not capture all the barriers PCPs experience in the referral and comanagement of patients with CKD with nephrologists, including the views of nurse practitioners and physician assistants who represent a growing proportion of primary care clinicians, as well as PCPs practicing in practice settings not as well 
represented in our study population (e.g., academic medical centers). However, the practice settings of PCPs in our study sample were similar in distribution to practice settings of PCPS nationally (private practice and multispecialty group practice: $85 \%$ vs. $88-93 \%$, respectively). ${ }^{44}$ Additionally, we aimed to capture perspectives of PCPs practicing in various settings and geographic locations; however, our study was not designed to compare differences between groups. Finally, we did not sample nephrologists and patients in our study. Studies of nephrologists and patients may also lead to further insights on key barriers and facilitators to effective referral and comanagement of patients with CKD. Studies addressing these weaknesses may inform the development, generalizability, and uptake of effective interventions.

In conclusion, PCPs identified several key challenges to effective co-management for patients with CKD that were also generalizable to other chronic illnesses. Development and greater adoption of effective collaborative tools facilitating timely adequate information exchange, clear delineation of roles and responsibilities between PCPs and specialists, and greater access to specialist advice may improve the quality, efficiency, and safety of patient care.

Corresponding Author: Raquel C. Greer, MD, MHS; Division of General Internal Medicine Johns Hopkins University, 2024 E. Monument Street, Suite 2-600, Baltimore, MD 21287, USA (e-mail: rgreer@jhmi.edu).

Funders This work was supported by the National Kidney Foundation of Maryland, the National Kidney Foundation, and the National Institutes of Health grant K23DK094975 (Greer), K23DK090304 (Abdel-Kader), and R01DK103935-01A1 (Cavanaugh). The content is solely the responsibility of the authors and does not necessarily represent the official views of the National Institutes of Health.

\section{Compliance with Ethical Standards:}

The Johns Hopkins Medicine Institutional Review Board approved the study protocol.

Conflict of Interest: The authors declare that they do not have a conflict of interest.

Publisher's Note: Springer Nature remains neutral with regard to jurisdictional claims in published maps and institutional affiliations.

\section{REFERENCES}

1. Go AS, Chertow GM, Fan D, McCulloch CE, Hsu CY. Chronic kidney disease and the risks of death, cardiovascular events, and hospitalization. N Engl J Med. 2004;351:1296-305.

2. US Renal Data System. USRDS 2016 Annual Data Report: Atlas of Chronic Kidney Disease and End-Stage Renal Disease in the United States. The National Institutes of Health, National Institute of Diabetes and Digestive and Kidney Disease Bethesda, MD. 2016.

3. Tonelli M, Wiebe N, Guthrie B, et al. Comorbidity as a driver of adverse outcomes in people with chronic kidney disease. Kidney Int. 2015;88:859-66.

4. Taylor EF, Lake T, Nysenbaum J, Perterson G, Meyers D. Coordinating care in the medical neighborhood: critical components and available mechanisms. White Paper (Prepared by Mathematica Policy Research under Contract No. HHSA290200900019I TO2). AHRQ Publication No.
11-0064. Rockville, MD: Agency for Healthcare Research and Quality. 2011.

5. Samal L, Wright A, Waikar SS, Linder JA. Nephrology co-management versus primary care solo management for early chronic kidney disease: a retrospective cross-sectional analysis. BMC Nephrol 2015;16:162.

6. Kidney Disease: Improving Global Outcomes (KDIGO) CKD Work Group. KDIGO 2012 Clinical Practice Guideline for the Evaluation and Management of Chronic Kidney Disease. Kidney International. 2013:1-150.

7. Jones C, Roderick $\mathbf{P}$, Harris $\mathbf{S}$, Rogerson $\mathbf{M}$. An evaluation of a shared primary and secondary care nephrology service for managing patients with moderate to advanced CKD. Am J Kidney Dis. 2006;47:103-14.

8. Haley WE, Beckrich AL, Sayre J, et al. Improving Care Coordination Between Nephrology and Primary Care: A Quality Improvement Initiative Using the Renal Physicians Association Toolkit. Am J Kidney Dis 2015;65:67-79

9. Levin A, Hemmelgarn B, Culleton B, et al. Guidelines for the management of chronic kidney disease. CMAJ 2008;179:1154-62.

10. Diamantidis CJ, Powe NR, Jaar BG, Greer RC, Troll MU, Boulware LE. Primary care-specialist collaboration in the care of patients with chronic kidney disease. Clin J Am Soc Nephrol. 2011;6:334-43.

11. O'Malley AS, Reschovsky JD. Referral and consultation communication between primary care and specialist physicians: finding common ground. Arch Intern Med. 2011;171:56-65.

12. Pham HH, O'Malley AS, Bach PB, Saiontz-Martinez C, Schrag D. Primary care physicians' links to other physicians through Medicare patients: the scope of care coordination. Ann Intern Med. 2009;150:23642.

13. Greer RC, Ameling JM, Cavanaugh KL, et al. Specialist and primary care physicians' views on barriers to adequate preparation of patients for renal replacement therapy: a qualitative study. BMC Nephrol. 2015; 16:37.

14. Greer RC, Crews DC, Boulware LE. Challenges perceived by primary care providers to educating patients about chronic kidney disease. J Ren Care. 2012;38:174-81.

15. Greer RC, Cooper LA, Crews DC, Powe NR, Boulware LE. Quality of patient-physician discussions about CKD in primary care: a crosssectional study. Am J Kidney Dis. 2011;57:583-91.

16. Hsieh H-F, Shannon SE. Three Approaches to Qualitative Content Analysis. Qual Health Res 2005; 15:1277-88.

17. Miles MB, Huberman AM, Saldana J. Qualitative Data Analysis: A Methods Sourcebook. 3th ed. Washington, DC: SAGE Publication.

18. Lo $\mathbf{C}$, Teede $\mathbf{H}$, Ilic $\mathbf{D}$, et al. Identifying health service barriers in the management of co-morbid diabetes and chronic kidney disease in primary care: a mixed-methods exploration. Fam Pract 2016;33:492-7.

19. Prouty CD, Mazor KM, Greene SM, et al. Providers' perceptions of communication breakdowns in cancer care. J Gen Intern Med. 2014;29:1122-30.

20. Dossett LA, Hudson JN, Morris AM, et al. The primary care provider (PCP)-cancer specialist relationship: A systematic review and mixedmethods meta-synthesis. CA Cancer J Clin. 2017;67:156-69.

21. Drawz PE, Archdeacon P, McDonald CJ, et al. CKD as a Model for Improving Chronic Disease Care through Electronic Health Records. Clin J Am Soc Nephrol. 2015;10:1488-99.

22. Navaneethan SD, Jolly SE, Sharp J, et al. Electronic health records: a new tool to combat chronic kidney disease? Clin Nephrol. 2013;79:17583.

23. Zimmerman DL, Selick A, Singh R, Mendelssohn DC. Attitudes of Canadian nephrologists, family physicians and patients with kidney failure toward primary care delivery for chronic dialysis patients. Nephrol Dial Transplant. 2003;18:305-9.

24. Pham HH. Good neighbors: How will the patient-centered medical home relate to the rest of the health-care delivery system? J Gen Intern Med 2010;25:630-4.

25. Beaulieu M, Levin A. Analysis of multidisciplinary care models and interface with primary care in management of chronic kidney disease. Semin Nephrol. 2009;29:467-74.

26. Chen AH, Yee HF Jr. Improving primary care-specialty care communication: lessons from San Francisco's safety net: comment on "Referral and consultation communication between primary care and specialist physicians". Arch Intern Med. 2011;171:65-7.

27. Vimalananda VG, Gupte G, Seraj SM, et al. Electronic consultations (econsults) to improve access to specialty care: a systematic review and narrative synthesis. J Telemed Telecare. 2015;21:323-30.

28. Chen AH, Kushel MB, Grumbach K, Yee HF Jr. Practice profile. A safety-net system gains efficiencies through 'eReferrals' to specialists. Health Aff (Millwood). 2010;29:969-71. 
29. National Kidney Disease Education Program. Collaborate with a Nephrol ogist. https://www.niddk.nih.gov/health-information/health-communication-programs/nkdep/identify-manage/manage-patients/nephrologist/Pages/nephrologist.aspx. Accessed 2 November 2018.

30. Mendu ML, McMahon GM, Licurse A, Solomon S, Greenberg J, Waikar SS. Electronic Consultations in Nephrology: Pilot Implementation and Evaluation. Am J Kidney Dis 2016;68:821-3.

31. Keely E, Li J, Magner P, Afkham A, Liddy C. Nephrology eConsults for Primary Care Providers: Original Investigation. Can J Kidney Health Dis. 2018;5:2054358117753619.

32. Tuot DS, Murphy EJ, McCulloch CE, Leeds K, Chan E, Chen AH Leveraging an electronic referral system to build a medical neighborhood. Healthc (Amst) 2015;3:202-8.

33. Gupte G, Vimalananda V, Simon SR, DeVito K, Clark J, Orlander JD. Disruptive Innovation: Implementation of Electronic Consultations in a Veterans Affairs Health Care System. JMIR Med Inform. 2016;4:e6.

34. Renal Physicians Association. Advanced CKD Patient Management Toolkit. http://www.renalmd.org/page/AdvancedCKDToolkit. Accessed 2 November 2018.

35. Lee MS, Ray KN, Mehrotra A, Giboney P, Yee HF Jr, Barnett ML. Primary Care Practitioners' Perceptions of Electronic Consult Systems: A Qualitative Analysis. JAMA Intern Med 2018;178:782-9.

36. Carrier E, Dowling MK, Pham HH. Care coordination agreements: barriers, facilitators, and lessons learned. Am J Manag Care. 2012;18:e398-404.

37. American College of Physicians. The Patient-Centered Medical Home Neighbor: The Interface of the Patient-Centered Medical Home with Specialty/Subspecialty Practices. Philadelphia: American College of Physicians. 2010
38. Mendu ML, Waikar SS, Rao SK. Kidney Disease Population Health Management in the Era of Accountable Care: A Conceptual Framework for Optimizing Care Across the CKD Spectrum. Am J Kidney Dis. 2017;70:122-31.

39. K/DOQI clinical practice guidelines for chronic kidney disease: evaluation, classification, and stratification. Am J Kidney Dis. 2002;39:S1-266.

40. Sperati CJ, Soman S, Agrawal V, et al. Primary Care Physicians' Perceptions of Barriers and Facilitators to Management of Chronic Kidney Disease: A Qualitative Study [Abstract]. J Am Soc Nephrol. 2016; 27: 840A.

41. Abdel-Kader K, Greer RC, Boulware LE, Unruh ML. Primary care physicians' familiarity, beliefs, and perceived barriers to practice guidelines in non-diabetic CKD: a survey study. BMC Nephrol. 2014;15:64.

42. Manca D, Varnhagen S, Brett-MacLean P, Allan GM, Szafran $\mathbf{0}$ Respect from specialists: concerns of family physicians. Can Fam Physician 2008;54: 1434-5.e14355.

43. Williams KM, Kirsh S, Aron D, et al. Evaluation of the Veterans Health Administration's Specialty Care Transformational Initiatives to Promote Patient-Centered Delivery of Specialty Care: A Mixed-Methods Approach. Telemed J E Health 2017;23:577-89.

44. Kane CK. Policy Research Perspectives: Updated Data on Physician Practice Arrangements: Physician Ownership Drops Below 50 Percent 2017. Accessed 2 November 2018.

Publisher's Note Springer Nature remains neutral with regard to jurisdictional claims in published maps and institutional affiliations. 